\section{Analysis of Gamma Ray Emissions From Fission Product Contributors to the Antineutrino Spectrum}

- C. J. Martoff, Samuel Kim, Temple University

- Michael Dion, David Glasgow, Oak Ridge National Laboratory
${ }^{235} \mathrm{U}$ Sample Irradiation at ORNL HFIR NAA Facility*

- Natural Uranium nitrate ICP calibration solution - 252.72 nano gram

- Irradiate for 30 seconds using PT-2, HFIR*, at

- "Rabbit" transit time = 20 seconds

142-Cs and 97-Yhave decayed away

- Gamma ray emission is measued for 30 seconds

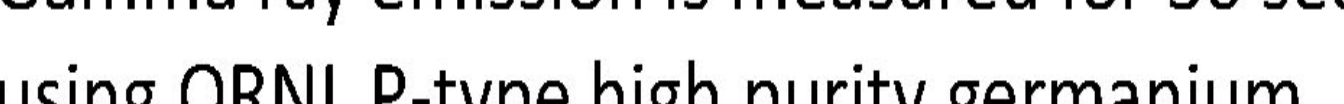

detector

*Oak Ridge National Laboratory (ORNL) *High Flux Isotope Reactor (HFIR)

*Neutron Activation Analysis facility (NAA)

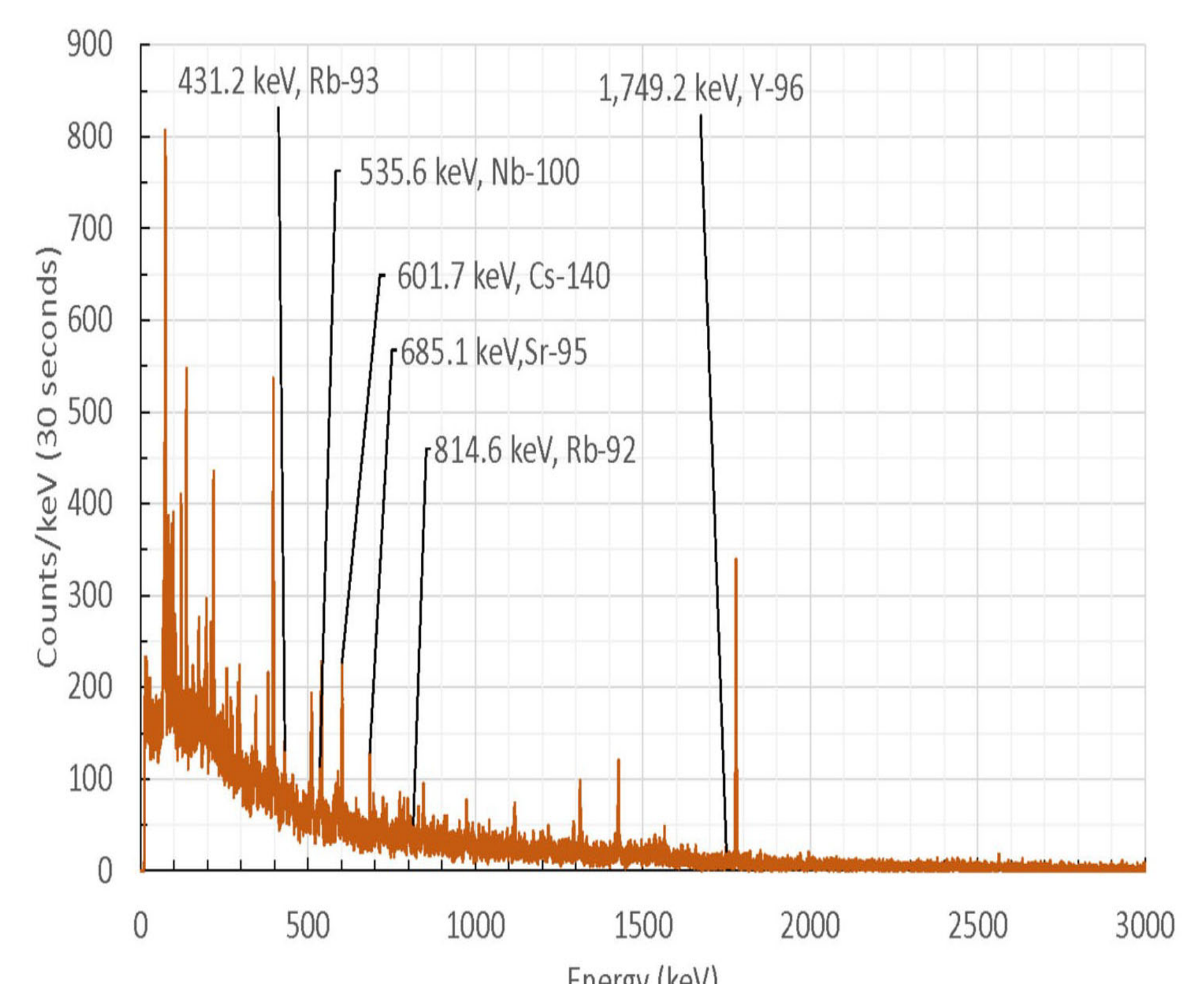
Reactor Neutrino ${ }^{1}$

- $\mathrm{A} 1 \mathrm{GW}$ thermal reactor emits approximately $1 \mathrm{E}+20$ antineutrinos per second

- A reactor produces over 1000 fission fragment nuclides, each of which beta decays.

Modeling the Reactor Neutrino Spectrum

Dwyer and Langford ${ }^{2}$

- Ab-initio summation method with nuclear data from ENDF/B-VII.1

(Positron energy at 4 to $6 \mathrm{MeV}$ )

$\begin{array}{llllll}-93-\mathrm{Rb} & (432.61 \mathrm{keV}, 5.84 \mathrm{~s}) & 100-\mathrm{Nb} & (535.67 \mathrm{keV}, 1.5 \mathrm{~s}) & 140-\mathrm{Cs} & (602.25 \mathrm{keV}, 63.7 \mathrm{~s})\end{array}$ $\begin{array}{llllll}-95-\mathrm{Sr} & (685.6 \mathrm{keV}, 23.9 \mathrm{~s}) & 92-\mathrm{Rb} & (814.98 \mathrm{keV}, 4.48 \mathrm{~s}) & 96-\mathrm{Y} & (1750.4 \mathrm{keV}, 5.34 \mathrm{~s})\end{array}$

- The tabulated Cumulative Fission Yields of these nuclides can be checked by their gamma ray emissions!

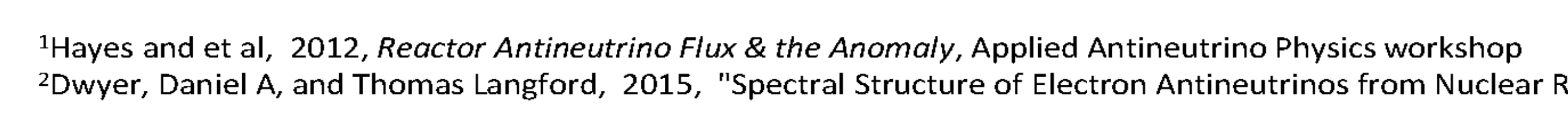

- Name given to an apparent bump in the energy spectrum of antineutrinos from reactors, around $5 \mathrm{MeV}$ energy

- From one fission, about 7 antineutrinos are produced.

.

\section{Expected Gamma Ray Rates}

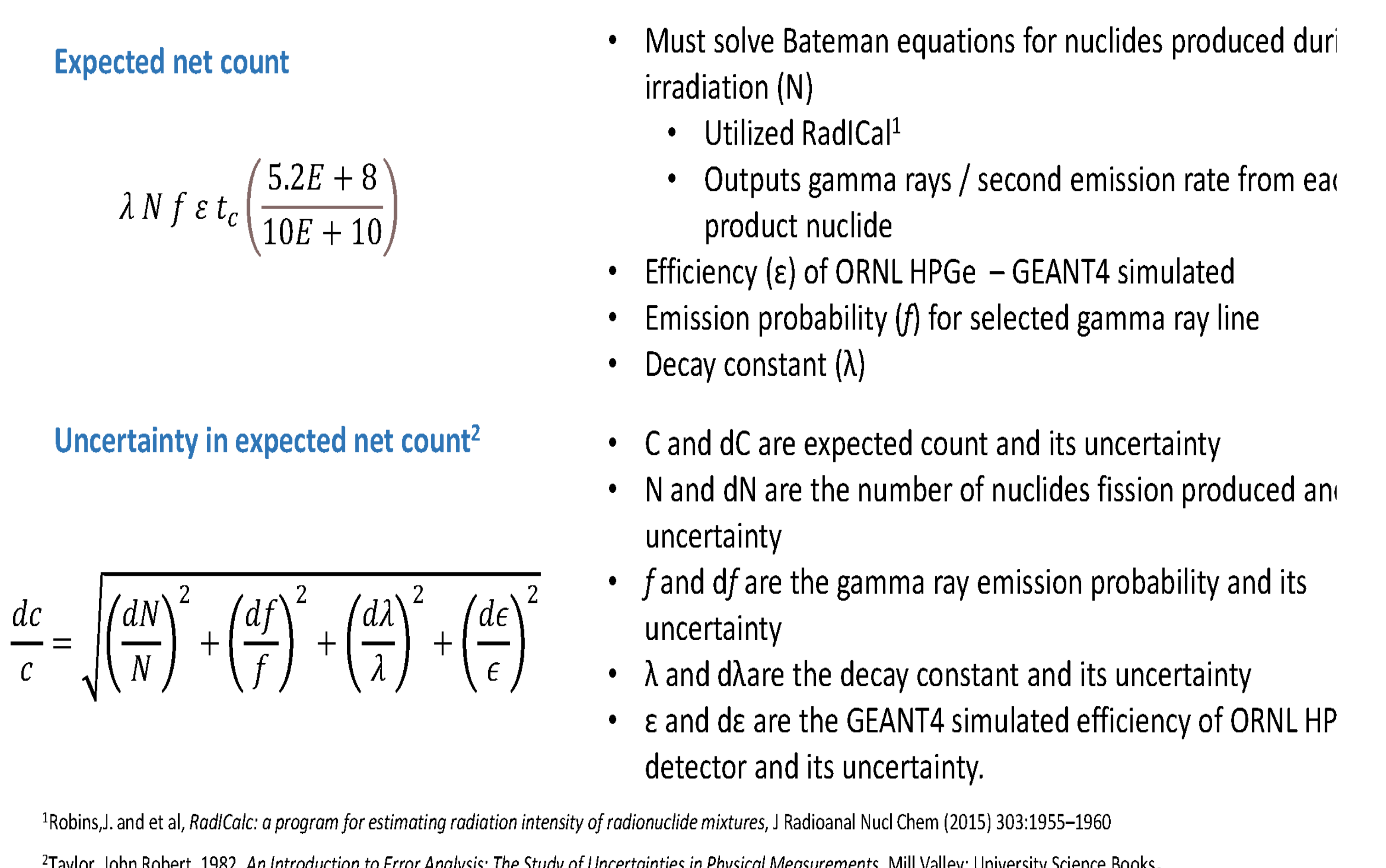

Nonlinear Peak Fitting (GNUPLOT)

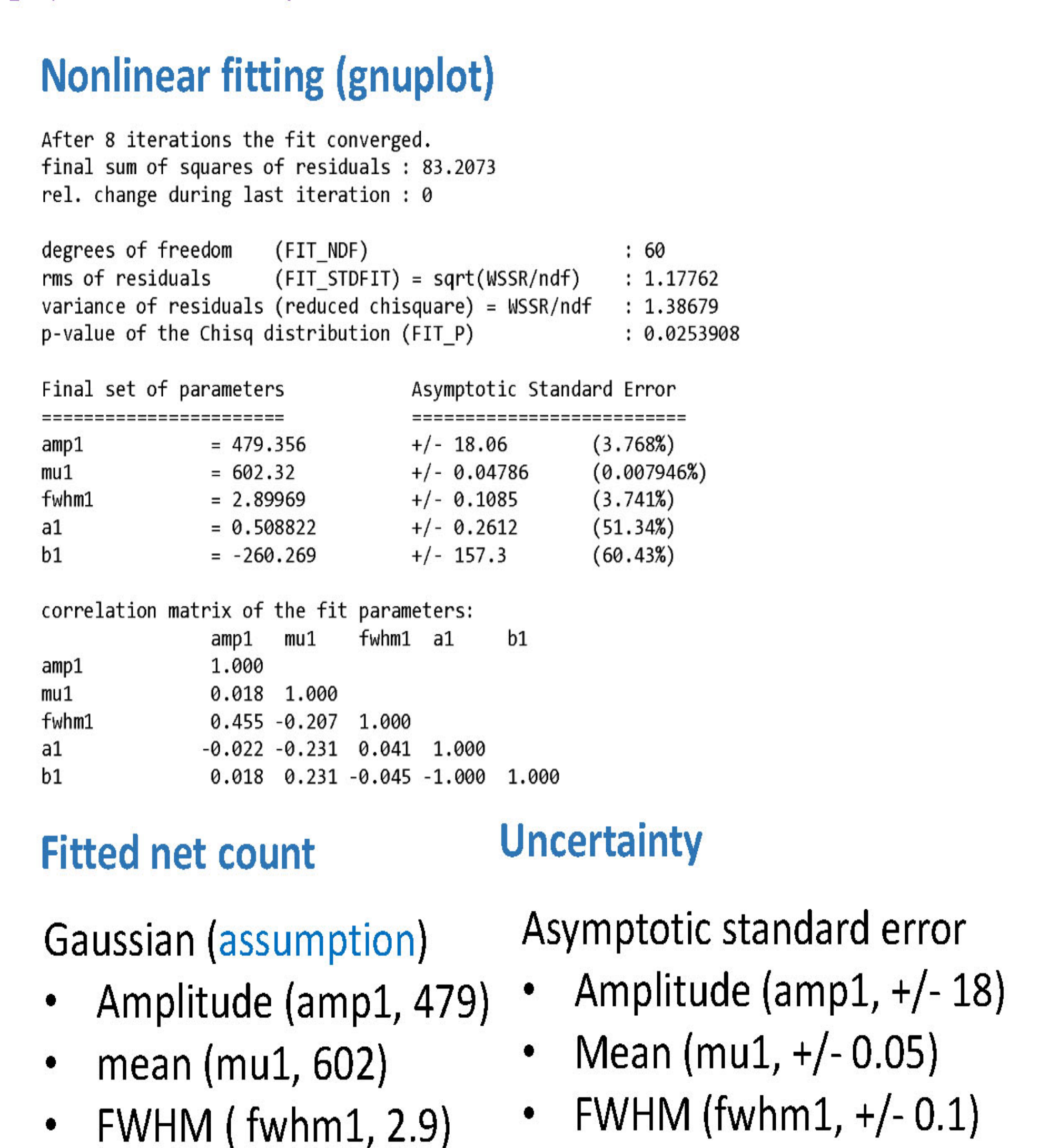

$\begin{array}{ll}\text { - mean (mu1, 602) } & \text { Mean (mu1, }+ \text { - }-0.05) \\ \text { - FWHM (fwhm1, 2.9) } & \text { - FWHM (fwhm1, +- - } 0.1)\end{array}$
Analysis of Observed Gamma Ray Peaks
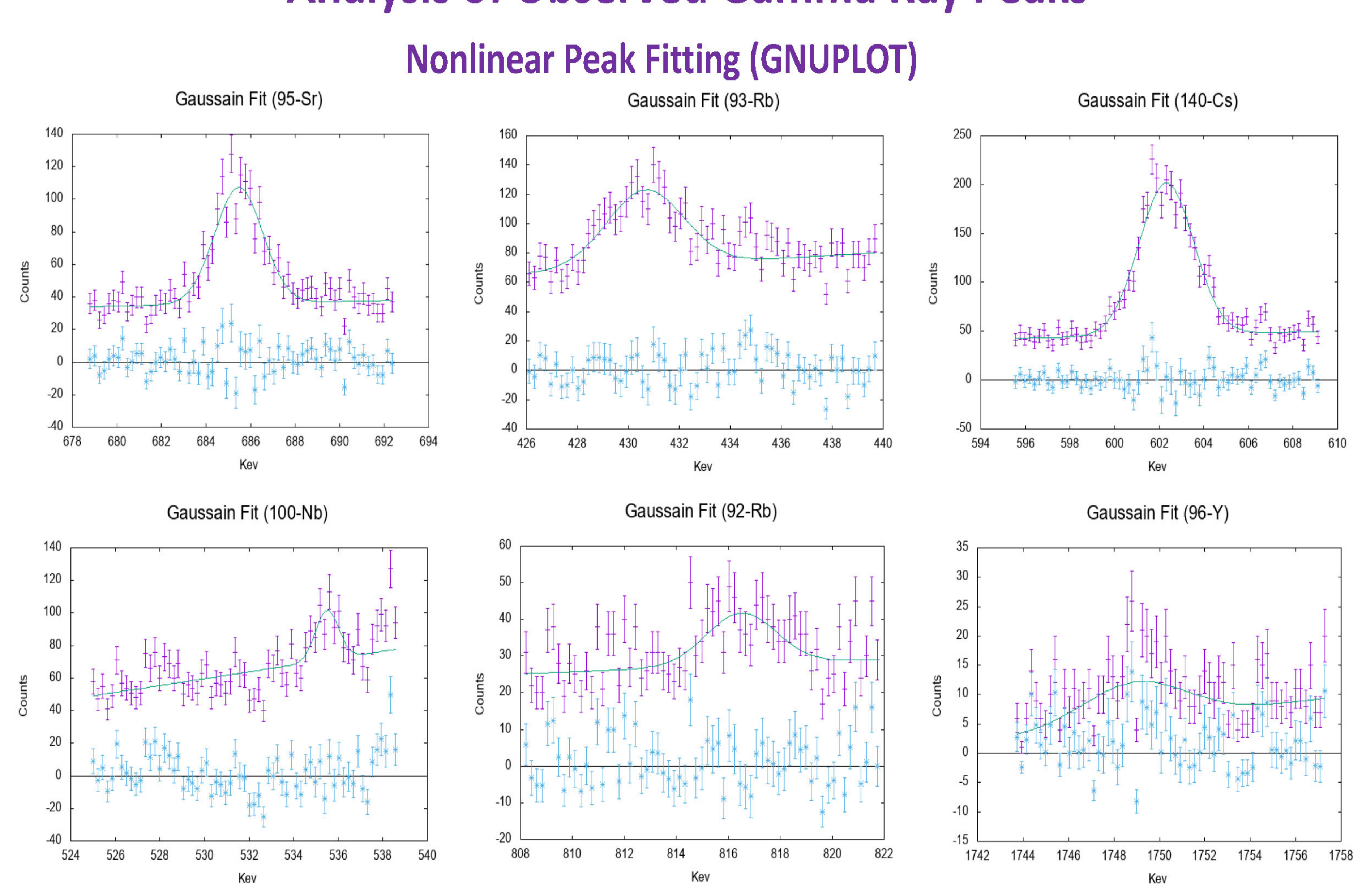

Analysis of Observed Gamma Ray Peaks

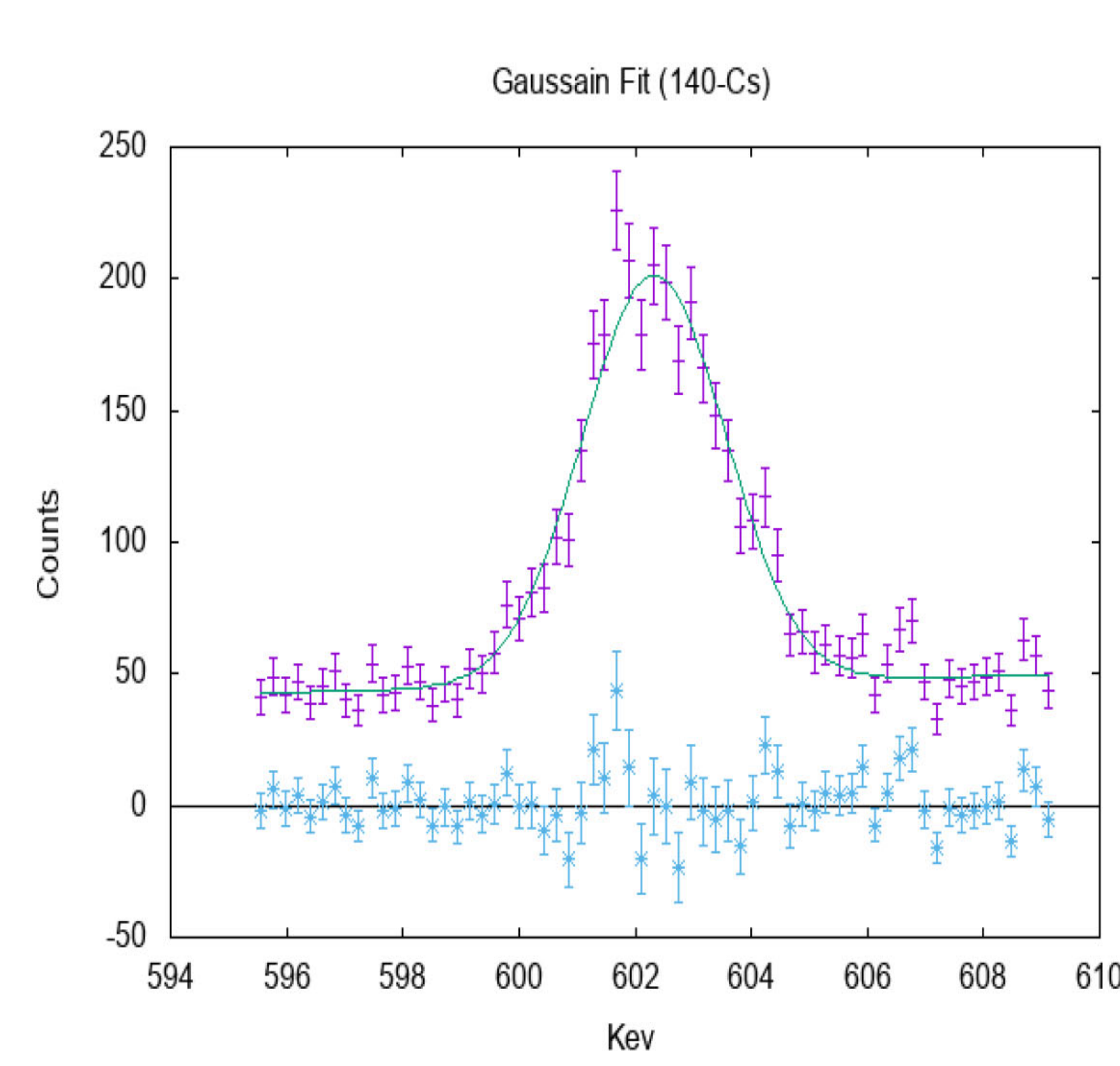

Alternate Analysis Method: Manual Peak Sum

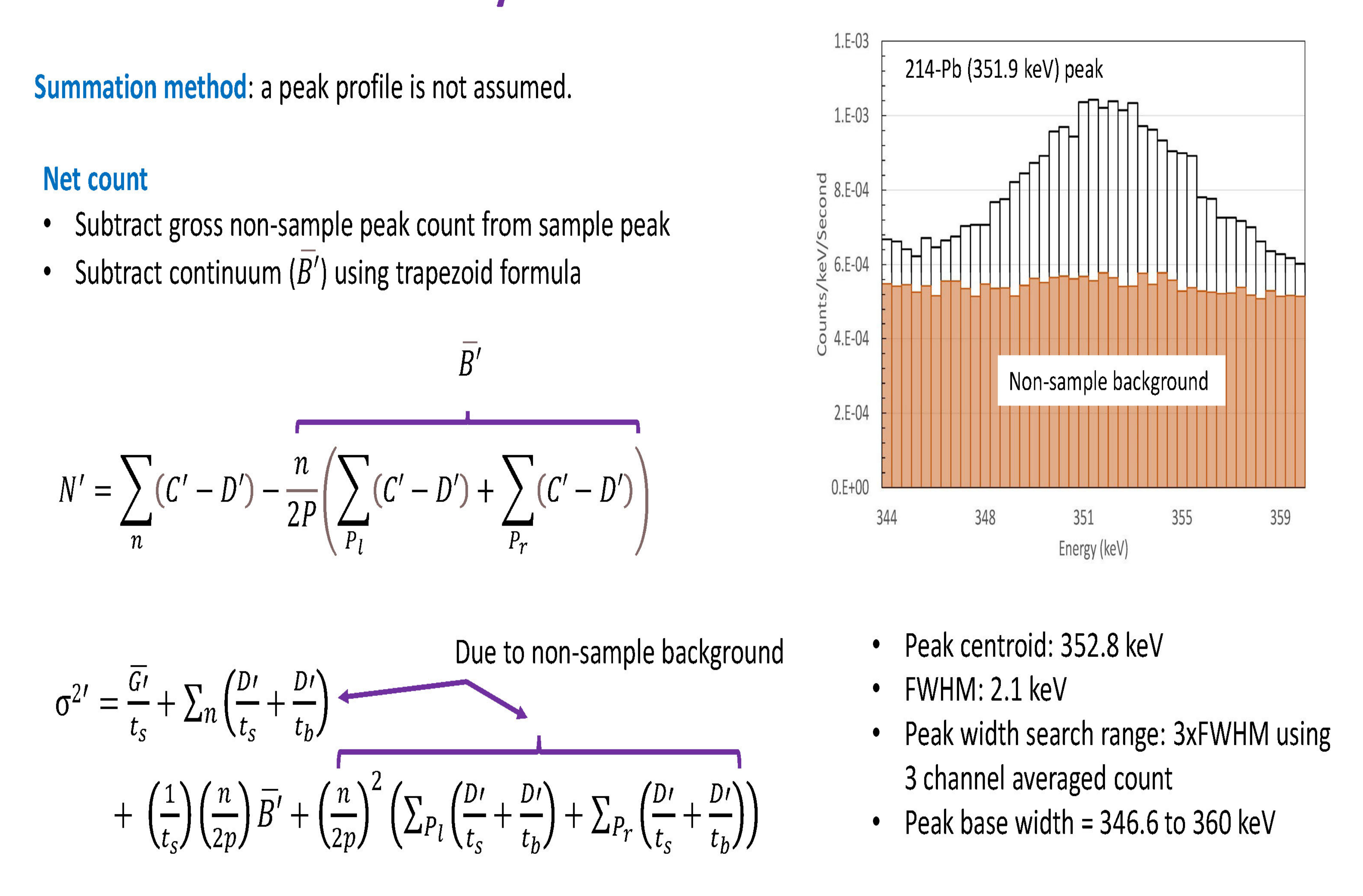

\section{Predicted and Measured Fission Daughter Counts}

95-Sr, 140-CS, 92-Rb and 96-Y

"Measurg rates within 2 of expected ate

93-Rb (low) and 100-Nb (high)

Measured rates are not within $2 \sigma$ expected

- rate

Partial support for Dwyer \& Langford

Follow Up

1) Errors in tabulated fission yields? Or in RadlCalc?

- RadlCal uses ENDF* VII, and Fitted and summed - RadlCal uses ENDF
use ENDF VIII

Better understanding about the systematic and random errors in RadlCal and analysis methods used. 3) Refine the calculation models 3) Further study is planned using more irradiations with larger samples at ORNL.

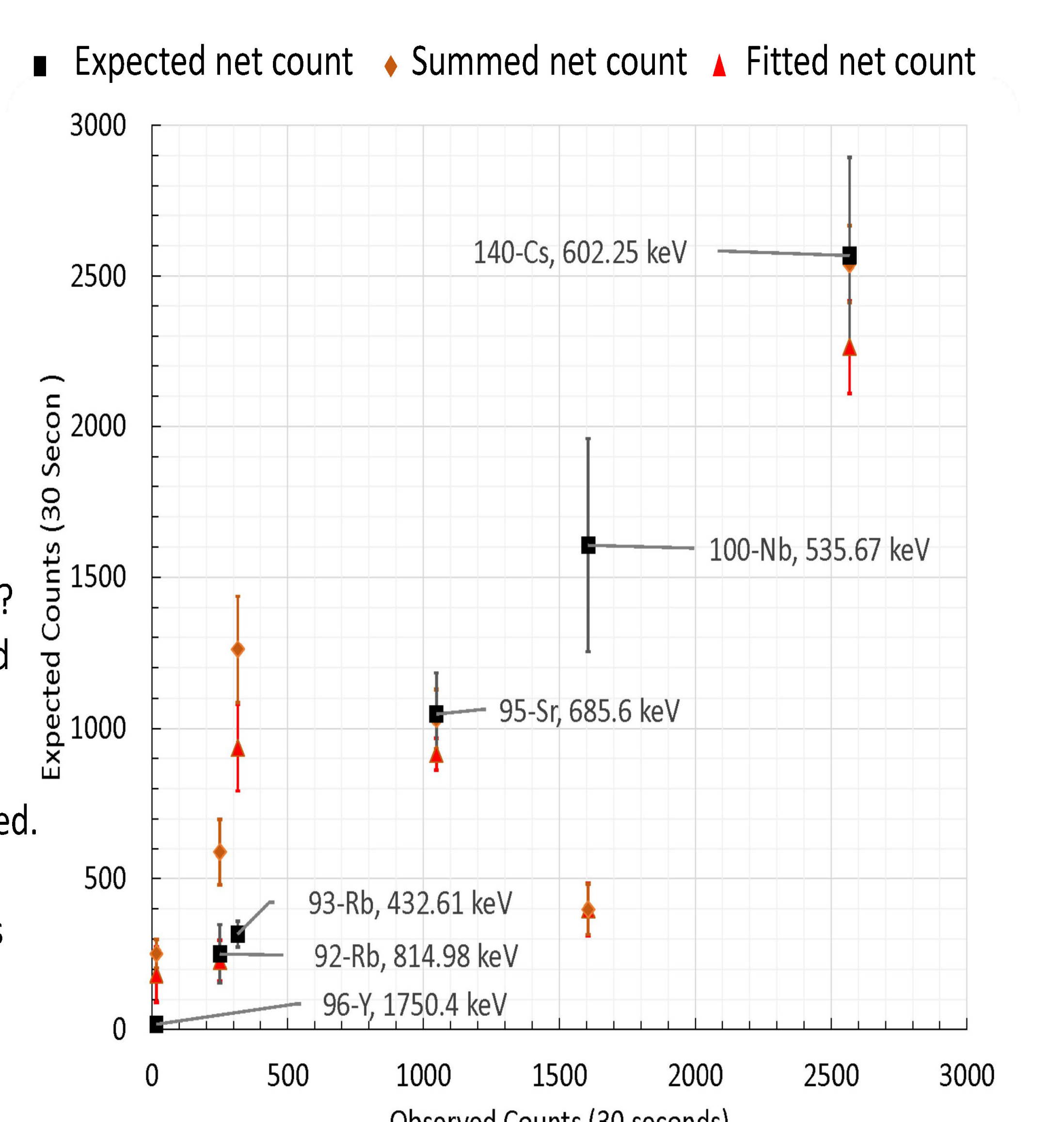

